\title{
Getting to the heart of the matter: detecting and managing cardiac complications in systemic sclerosis
}

\author{
John Varga (1) , ${ }^{1}$ Daniel C Lee ${ }^{2}$
}

Cardiac complications of systemic sclerosis (SSc) are well-deservedly gaining the attention of the medical community. This increased interest is driven by technological advances in non-invasive imaging technologies such as tissue Doppler echocardiography and cardiac MR (CMR) that reveal frequent involvement of the heart and its clinical importance in SSc. Historically, autopsy studies in SSc indicate a high prevalence $(>50 \%)$ of cardiac fibrosis, ${ }^{1}$ and clinically evident cardiac involvement has long been considered a poor prognostic marker in SSc. ${ }^{2}$ Indeed, the European Clinical Trials and Research Group reported that $26 \%$ of SSc disease-related mortality could be attributed to cardiac causes. ${ }^{4}$ However, the overall prevalence of cardiac disease in SSc is unknown, in part due to the lack of a consensus definition, classification of 'subclinical' cardiac involvement, and the plethora of diverse approaches employed for its detection. ${ }^{5}$

The cardiac complications of SSc encompass multiple distinct entities including primary cardiac involvement manifested by diastolic dysfunction (an early manifestation of cardiac fibrosis), heart failure with preserved ejection faction, conduction blocks and arrhythmias, myocarditis, as well as pericardial disease; and cardiac involvement secondary to systemic or pulmonary arterial hypertension, renal failure, amyloidosis and other SSc complications. The pathogenesis of SSc-associated primary cardiac involvement is not well understood, and likely encompasses small vessel damage, vasoconstriction and chronic ischaemia-reperfusion injury, cardiac inflammation and fibrosis.

Although systolic dysfunction in SSc is uncommon, diastolic dysfunction is common and predicts poor outcomes. In an observational study of 153 consecutive SSc patients, we showed that 23\% had

\section{${ }^{1}$ Northwestern Scleroderma Program, Chicago, Illinois, USA \\ ${ }^{2}$ Division of Cardiology, Northwestern University - Chicago, Chicago, Illinois, USA}

Correspondence to Dr John Varga, Medicine, Northwestern University, Chicago, IL 60611, USA; j-varga@northwestern.edu echocardiographically defined left ventricular diastolic dysfunction (often asymptomatic), and its presence was predictive of mortality. ${ }^{6}$ A recent study in a large and unselected cohort of SSc patients similarly found that the incidence of diastolic dysfunction was $17 \%$ at baseline, and increased to $29 \%$ during follow-up of 3.4 years. ${ }^{7}$ Significantly, mortality in this group was increased more than fourfold compared with that in SSc patients without evidence of diastolic dysfunction at baseline, underscoring the clinical significance of diastolic dysfunction, and the need for its early recognition.

The prevalence of cardiac involvement in SSc is even higher when screening using CMR. In a study of 62 SSc patients with no prior heart disease or coronary artery disease (CAD) risk factors, $45 \%$ had myocardial fibrosis assessed by late gadolinium-enhanced CMR. ${ }^{8}$ Moreover, despite the absence of significant epicardial coronary stenosis by CT coronary angiography, stress CMR perfusion imaging revealed subendocardial perfusion defects (a sign of impaired microvascular perfusion) in $79 \%$ of the patients. In a prospective study of 201 patients with SSc without known cardiac involvement, cardiac fibrosis was detected by late gadolinium enhancement CMR in $27.9 \%$, most of whom had no evidence of cardiac abnormalities by echocardiography. ${ }^{9}$ The presence of late gadolinium enhancement was correlated by ventricular arrhythmias. Even without late gadolinium enhancement, the extracellular volume (ECV) fraction (a CMR index of diffuse myocardial fibrosis) is higher in SSc patients compared with controls. ${ }^{10}$ These observations provide strong evidence of the high prevalence of clinical — and subclinical—cardiac involvement in SSc patients.

Given the high prevalence and clinical impact of cardiac involvement in SSc, deeper understanding of its natural history and treatment are sorely needed. In this issue of Annals of the Rheumatic Diseases, Valentini et al present findings from the DeSScipher (to decipher the optimal treatment of SSc) cohort study evaluating the impact of aspirin and vasodilator therapy on myocardial disease in SSc. ${ }^{11}$ This large multicentre observational and event-driven study enrolled 654 SSc patients from 20 sites. Patients underwent thorough cardiac monitoring (exceeding current clinical practice), including history and physical exam at baseline and every 3 months, along with ECG, Holter and echocardiographic evaluations at baseline and every 6 months. Patients were not preselected for cardiac involvement, so the population represents a primary prevention cohort. Patients were classified as receiving vasodilator therapy if they received an ACE inhibitor, an angiotensin II receptor blocker or a calcium channel blocker in some combination. Some $20 \%$ also received targeted vasodilator therapy (prostanoids, endothelin receptor antagonists and phosphodiesterase type 5 inhibitors). Outcome measures included occurrence of (1) ventricular arrhythmias (considered a sign of myocardial ischaemia), (2) Q waves, cardiac blocks and/or pacemaker implantation (considered a sign of fibrosis) and (3) left ventricular ejection fraction $<55 \%$ and/or congestive heart failure (considered a sign of progressive myocardial disease). The results showed that while vasodilator therapy was not associated with measured outcomes on univariate analysis, on multivariate analysis vasodilator treatment was associated with lower incidence of ventricular arrhythmia. Additionally, low-dose aspirin was associated with a lower incidence of the combined endpoint $(\mathrm{Q}$ waves, conduction blocks and/or pacemaker implantation) in univariate and multivariate analysis.

These important results need to be placed in the context of the limitations of the study. Foremost is the observational nature of the study. Assignment to treatment groups was not randomised, raising the distinct possibility that unmeasured differences between the treatment groups may have confounded the results. Moreover, the outcome measures selected were not standard for a cardiac outcome study, and the relatively low incidence of individual outcomes required combination into groups based on presumed (although not proven) pathophysiology. Nonetheless, the study raises interesting and timely questions regarding the management of SSc-associated cardiac disease.

First, should low-dose aspirin be routinely prescribed to SSc patients? The mechanism by which $\mathrm{Q}$ waves (the sine qua non for diagnosing myocardial infarction by ECG) are prevented by aspirin would presumably be via platelet inhibition or its anti-inflammatory properties. However, for the primary prevention of CAD in non-SSc cohorts, recent large trials and meta-analyses have demonstrated that the benefits of ASA do not outweigh bleeding risks except 
for in high-risk individuals, for example, those with 10-year atherosclrotic cardiovascular disease risk $>15 \% .{ }^{12}$ The limited data on ischaemic events in SSc suggest that incidence is higher than in matched controls, but the 10-year risk of ischaemic events is likely $<10 \% .{ }^{13} 14$ Cardiac blocks and pacemaker implantation are signs of conduction system disease, which in SSc patients is more commonly due to fibrosis of the conduction system but could also be due to ischaemic injury. Did the patients in this study actually have myocardial scar? Because the characteristic patchy, mid-myocardial fibrosis of SSc detected by CMR rarely causes wall motion abnormalities, routine echocardiography is not sufficient to detect its presence.

What about vasodilator therapy? Autopsy studies of SSc patients have demonstrated a high prevalence of contraction band necrosis (a sign of ischaemic injury) despite the absence of epicardial coronary artery disease. ${ }^{1}$ The presumed mechanism of ischaemic injury is either vasospasm or small vessel disease. This provides a pathophysiological basis for the potential benefit of vasodilators for prevention of myocardial fibrosis in SSc. The type of ventricular arrhythmias seen in DeSScipher were not sufficiently characterised, but most ventricular arrhythmias can be either ischaemia or scar mediated. The heterogeneous combinations of medications in the vasodilator group makes translation of findings from DeSScipher into clinical recommendations difficult. However, perfusion and scar imaging might help to define the pathophysiological basis of this potential treatment benefit.

These concerns notwithstanding, the DeSScipher investigators should be lauded for embarking on this large, prospective, multicentre observational study of cardiac outcomes in SSc. The results of this study will surely provide more insights into the natural history of cardiovascular complications with continued follow-up of the patients. Hopefully, these observations will inspire further studies of cardiac involvement in SSc, and offer lessons that will enhance future investigations. Although randomised controlled trials evaluating hard events (eg, myocardial infarction, cardiac death) are ultimately required to establish safety and efficacy of therapy, such studies require large numbers and/or long-term follow-up. Prior to embarking on large and expensive pivotal trials, animal studies or smaller patient studies using more sensitive surrogate endpoints may help to better understand underlying biology, and identify the agent (or combination) with maximum therapeutic potential. For example, in a recent study of 44 SSc patients evaluated for haematopoietic stem cell transplantation (HSCT), serial measurement of diffuse myocardial fibrosis by CMR demonstrated significant progression of ECV fraction in patients who did not undergo HSCT, while significant regression was noted in those who did receive HSCT. Furthermore, the changes in ECV were mirrored by changes in modified Rodnan Skin Score. ${ }^{15}$ These observations highlight the utility of CMR, and also demonstrate that cardiac fibrosis in SSc is potentially reversible.

Multiple recent studies underscore the high prevalence and significant clinical impact of cardiac involvement in SSc- but also uncover unmet needs. Consensus guidelines for screening, utilisation of advanced imaging and the appropriate management of the entire spectrum of SSc cardiac involvement-from subclinical to overt, from the myocardium to the conduction system to the pericardium - are emerging, but to date are largely based on expert opinion. ${ }^{16} 17$ To inform these guidelines, we urge more investigation in this area. Needed are basic research employing animal models of disease to understand the pathogenesis of SSc cardiac disease and identify potential therapeutic targets, and clinical research to understand the risk factors, predictors, natural history and relevant biomarkers of cardiac involvement (including both blood and imaging) for patient stratification and monitoring. Ultimately, controlled clinical trials will be required in order to demonstrate safety and efficacy of novel drugs and treatment strategies for SSc-associated cardiac involvement.

\section{Handling editor Josef S Smolen}

Contributors JV: autoharp. DCL: coauthor.

Funding The authors have not declared a specific grant for this research from any funding agency in the public, commercial or not-for-profit sectors.

Competing interests None declared.

Patient consent for publication Not required.

Provenance and peer review Not commissioned; internally peer reviewed.

(C) Author(s) (or their employer(s)) 2019. No commercial re-use. See rights and permissions. Published by BMJ.

\section{Check for updates}

To cite Varga J, Lee DC. Ann Rheum Dis 2019;78:1452-1453.

Received 27 September 2019

Accepted 30 September 2019

Published Online First 3 October 2019

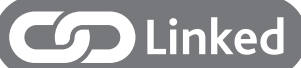

- http://dx.doi.org/10.1136/annrheumdis-2019215486

Ann Rheum Dis 2019;78:1452-1453. doi:10.1136/annrheumdis-2019-216115

\section{ORCID iD}

John Varga http://orcid.org/0000-0001-8400-687X

\section{REFERENCES}

1 Bulkley BH, Ridolfi RL, Salyer WR, et al. Myocardial lesions of progressive systemic sclerosis. A cause of cardiac dysfunction. Circulation 1976;53:483-90.

2 D'Angelo WA, Fries JF, Masi AT, et al. Pathologic observations in systemic sclerosis (scleroderma). A study of fifty-eight autopsy cases and fifty-eight matched controls. Am J Med 1969;46:428-40.

3 Follansbee WP, Miller TR, Curtiss El, et al. A controlled clinicopathologic study of myocardial fibrosis in systemic sclerosis (scleroderma). J Rheumatol 1990;17:656-62.

4 Tyndall AJ, Bannert B, Vonk $\mathrm{M}$, et al. Causes and risk factors for death in systemic sclerosis: a study from the EULAR scleroderma trials and research (EUSTAR) database. Ann Rheum Dis 2010;69:1809-15.

5 Desai CS, Lee DC, Shah SJ. Systemic sclerosis and the heart: current diagnosis and management. Curr Opin Rheumatol 2011;23:545-54.

6 Hinchcliff M, Desai CS, Varga J, et al. Prevalence, prognosis, and factors associated with left ventricular diastolic dysfunction in systemic sclerosis. Clin Exp Rheumatol 2012;30:\$30-7

7 Tennøe AH, Murbræch K, Andreassen JC, et al. Left ventricular diastolic dysfunction predicts mortality in patients with systemic sclerosis. J Am Coll Cardiol 2018;72:1804-13.

8 Rodriguez-Reyna TS, Morelos-Guzman M, HernandezReyes $\mathrm{P}$, et al. Assessment of myocardial fibrosis and microvascular damage in systemic sclerosis by magnetic resonance imaging and coronary angiotomography. Rheumatology 2015;54:647-54.

9 Gargani L, Todiere G, Guiducci S, et al. Early Detection of Cardiac Involvement in Systemic Sclerosis: The Added Value of Magnetic Resonance Imaging. JACC Cardiovasc Imaging 2019;12:927-8.

10 Lee DC, Hinchcliff ME, Sarnari R, et al. Diffuse cardiac fibrosis quantification in early systemic sclerosis by magnetic resonance imaging and correlation with skin fibrosis. J Scleroderma Relat Disord 2018:3:159-69.

11 Valentini G, Huscher D, Riccardi A, et al. Vasodilators and low-dose acetylsalicylic acid are associated with a lower incidence of distinct primary myocardial disease manifestations in systemic sclerosis: results of the DeSScipher inception cohort study. Ann Rheum Dis 2019;78:1576-82.

12 Chiang KF, Shah SJ, Stafford RS. A practical approach to low-dose aspirin for primary prevention. JAMA 2019:322.

13 Chu S-Y, Chen Y-J, Liu C-J, et al. Increased risk of acute myocardial infarction in systemic sclerosis: a nationwide population-based study. Am J Med 2013;126:982-8

14 Man A, Zhu Y, Zhang Y, et al. The risk of cardiovascular disease in systemic sclerosis: a population-based cohort study. Ann Rheum Dis 2013;72:1188-93.

15 Lee DC, Shah SJ, Benefield BC, et al. Abstract 17650 : myocardial fibrosis in systemic sclerosis, progression and response to therapy measured by cardiovascular magnetic resonance. Anaheim, California: Circulation, 2017: 136. A17650.

16 Bissell L-A, Anderson M, Burgess M, et al. Consensus best practice pathway of the UK systemic sclerosis Study group: management of cardiac disease in systemic sclerosis. Rheumatology 2017;56:912-21.

17 Farge D, Burt RK, Oliveira M-C, et al. Cardiopulmonary assessment of patients with systemic sclerosis for hematopoietic stem cell transplantation: recommendations from the European Society for blood and marrow transplantation autoimmune diseases Working Party and collaborating partners. Bone Marrow Transplant 2017;52:1495-503. 